\title{
LA LUTTE CONTRE LES MARINGOUINS
}

630.4

$\mathrm{C} 212$

P 936

1968

fr.

c. 3 


\section{ÉTAPES PRINCIPALES}

- Prévoir la campagne bien avant l'arrivée des maringouins.

- Dresser à l'avance un relevé ef la carte du terrain. S'assurer de la main-d'oeuvre et des matériaux nécessaires pour le début de la saison de reproduction.

- Éliminer les foyers de reproduction saisonniers ou permanents, c'est-d̀-dire assainir les marécages, combler les dépressions, endiguer les plaines d'alluvions et enlever tout récipient artificiel.

- Épandre les produits chimiques sur les nappes d'eau avant que les larves se changent en nymphes.

- Détruire d'abord les foyers de reproduction et les larves; s'attaquer aux maringouins adultes en dernier ressort.

- Utiliser seulement des produits recommandés et suivre le mode d'emploi indiqué.

- Éviter la contamination des cultures et des eaux courantes.

- Conserver sur une carte à grande échelle l'emplacement des foyers de reproduction. Si possible, retenir les mêmes employés chaque année. L'expérience personnelle et des fiches tenues à jour rendront chaque année la campagne plus efficace. 


\title{
LA LUTTE CONTRE LES MARINGOUINS
}

\author{
L. Colin Curtis \\ Station de recherches, Kamloops, \\ Colombie-Britannique
}

\section{POURQUOI DES CAMPAGNES CONTRE LES MARINGOUINS}

Quelques-uns des plus vastes et plus fertiles terrains de reproduction de maringouins se trouvent au Canada: nos étangs et marais en abritent près de 60 espèces différentes. Comme la plupart des maringouins ont besoin de sang pour se nourrir, pondre et survivre, ils constituent un véritable fléau.

Dans beaucoup de pays, aux tropiques en particulier, les maringouins (ou moustiques) transmettent des maladies dont les plus connues sont la malaria et la fière jaune. Au Canada il est heureux qu'il n'y ait qu'une maladie grave attribuable aux maragouins: la maladie du sommeil du cheval. Elle peut se communiquer a l'homme mais dans la plupart des régions du pays elle est très rare.

Même s'ils ne sont qu'une cause négligeable de maladies, les maringouins infligent chaque année au Canada des pertes incalculables de temps et d'argent. Non contents de troubler le sommeil ou les plaisirs du campeur, du pêcheur ou du piqueniqueur, ils sont une source sérieuse d'ennuis pour le cultivateur: sous leurs assauts violents, le bovin perd du poids, la vache donne moins de lait, le cheval de trait devient intraitable et les poules diminuent leur ponte. II est même arrivé à des ouvriers employés à la récolte d'être chassés par les maringouins des vergers, des plantations de petits fruits ou des prairies d foin. Ces insectes ont forcé l'arrêt du travail dans les chantiers d'abattage forestier ou de construction, et cela a grands frais pour les entrepreneurs. Ces raisons et d'autres encore jus. tifient les dépenses et le travail consacrés aux campagnes contre les maringouins.

\section{BIOLOGIE}

Le cycle évolutif du maringouin comporte quatre phases: l'oeuf, la larve, la nymphe et l'adulte.

Les oeufs fraíchement pondus ont la forme de cigares de $1 / 16 \mathrm{e}$ de pouce. D'abord pâles, ils virent au noir dès leur exposition a l'air et a la lumière. IIs sont pondus a la surface de l'eau ou au sol danś les endroits humides.

La larve est ce petit "ver" frétillant que l'on voit souvent dans les tonneaux a pluie. II se nourrit des infimes particules, végétales ou animales, en suspension dans l'eau et se développe en une série de mues au cours desquelles sa taille augmente graduellement. Après quatre mues, la larve se change en nymphe.

La nymphe ressemble à une 


\section{绿}

Oeufs

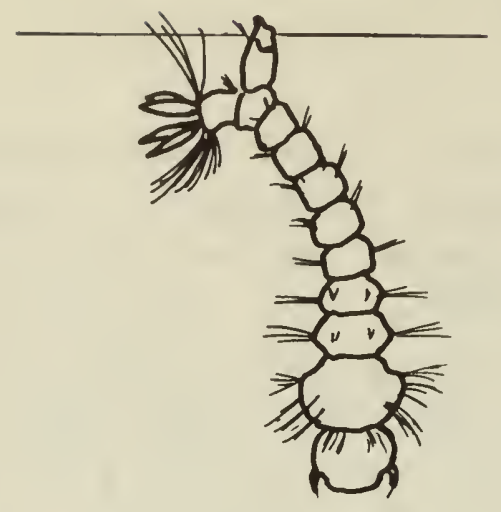

Larve

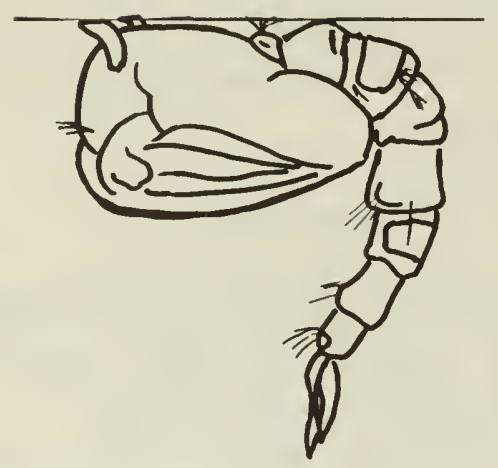

Pupe

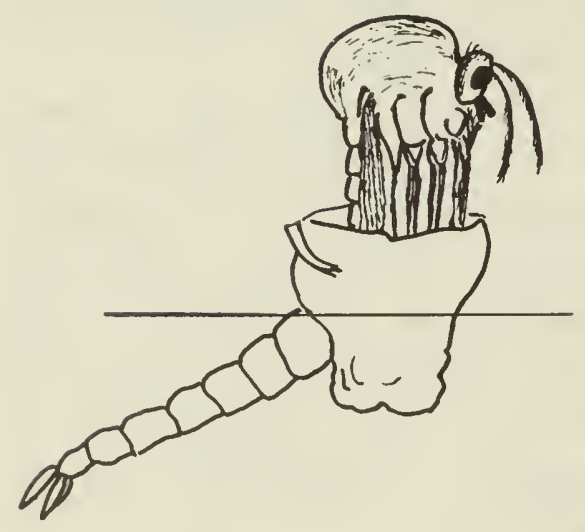

Adulte sortant de la pupe virgule noire. C'est elle qui abrite la phase de maturation de l'insecte adulte. Après quelques jours, elle remonte à la surface de l'eau, sa peau se déchire le long du dos et le maringouin adulte en sort lentement. II s'appuie sur l'enveloppe maintenant vide afin d'affermir ses pattes et ses ailes, puis il prend son vol et va se poser dans un endroit humide, à l'ombre du feuillage.

Pendant quelques jours les adultes des deux sexes se nourrissent du nectar des fleurs, après quoi ils s'accouplent. Le mâle meurt bientôt après mais la femelle part en quête de sang.

Une fois gorgée de sang, la femelle se repose encore quelques jours puis elle dépose ses oeufs a un endroit propice. Chez la plupart des espèces, la femelle ne survit pas longtemps à la ponte, mais chez certaines, elle a la propriété de recommencer à plusieurs reprises le le cycle du sucement de sang et de la ponte.

Tous les maringouins passent par les phases décrites plus haut mais ils varient par la répartition des phases selon les saisons.

\section{HIVERNEMENT Ȧ L'ÉTAT D'OEUF}

La plupart des maringouins communs au Canada pondent en automne sur le sol ou sur des racines d'herbe à proximité de l'eau. Au printemps, lorsque le sol est submergé, les larves ne tardent pas à éclore et en l'espace d'une à trois semaines, elles traversent les phases larvaires et nymphales pour arriver au stade adulte. La plupart des oeuts hivernant au sol ont besoin 
pour éclore d'une alternance de périodes d'assèchement et de refroidissement, mais même avec cela, ils ne réussiront pas tous à éclore à la première submersion; des submersions successives au cours d'une même saison donneront plusieurs séries d'éclosions. On a compté ¡usqu'à 3,218 larves sur une plaque de gazon de 8 pouces de diamètre.

Du fait qu'il leur faut des changements périodiques du niveau de l'eau, les moustiques se retrouvent dans les plaines alluviales des rivières, les étangs formés par la fonte des neiges ou les pluies printanières, l'eau qui s'infiltre derrière les digues ou encore l'excédent d'eau d'irrigation. Quelques-unes des espèces les plus gênantes croissent dans les marais côtiers.

\section{HIVERNEMENT AU STADE ADULTE}

En moins grand nombre au Canada sont les espèces qui hivernent à l'état adulte dans des lieux abrités comme les caves, les terriers et les arbres creux. Au printemps, après un repas de sang, les femelles déposent leurs oeufs dans l'eau, soit un par un, soit en petites masses flottantes. Ces oeufs qui ne tolèrent ni le gel ni l'assèchement, éclosent peu après et en sai son chaude il peut s'en produire plusieurs générations avant les gelées d'automne. Vers la fin de l'été, une génération d'adultes arrivent à terme; après l'accouplement le mâle meurt tandis que la femelle se met à l'abri pour l'hiver, perpétuant ainsi le cycle.

Les maringouins de ce type se reproduisent dans les étangs et les marécages de même que dans un vaste choix de récipients artificiels: bassins ornementaux, étangs de sédimentation, bassins collecteurs des eaux de rue, toits plats refroidis à l'eau, tonneaux à pluie, abreuvoirs à chevaux, vieux pneus, bouteilles ef boîtes en fer-blanc vides, vases funéraires, tonneaux à incendie disposés le long des voies de chemin de fer, puisards et fosses septiques. Une espèce canadienne au moins ne se reproduit pratiquement que dans les arbres creux.

\section{LE PLAN D'ATTAQUE}

C'est au printemps ou au début de l'été qu'il faut combattre les maringouins, au moment où les larves se développent. Préparer la campagne dès l'été précédent.

II est préférable que plusieurs municipalités joignent leurs efforts pour une campagne unique. Les maringouins ne reconnaissent ni clôtures ni frontières municipales; certains peuvent parcourir des milles de distances, et ce n'est pas toujours à l'endroit de leur éclosion qu'ils sont les plus gênants. Aussi, les bons résultats d'une compagne sont proportionnels à la grandeur de l'étendue couverte.

Dans une région la campagne peut être mise sur pied par les autorités ou un comité spécial. Respecter les lois provinciales sur la lutte contre les maringouins et les autres insectes nuisibles et recourir aux conseils d'un entomologiste provincial ou du ministère de la Santé publique.

Choisir des ouvriers qui connaissent à fond la région et sur 
qui on pourra compter chaque année. Le succès des travaux reposera pour une large part sur la connaissance personnelle des foyers de reproduction.

\section{RELATIONS PUBLIQUES}

La lutte contre les maringouins est essentiellement une entreprise communautaire et son plein succès exige l'appui de toute la population. Obtenir l'aide de la presse, de la radio, des clubs sociaux et des chambres de commerce. Éviter de froisser les propriétaires, les agriculteurs et tous ceux dont la bonne volonté est essentielle au succès de la campagne. En effectuant les inspections ou les traitements, fermer les barrières, remettre en ordre les clôtures brisées et laisser en paix les animaux de ferme; avertir les apiculteurs 48 heures avant le début des pulvérisations. Se souvenir que dans la plupart des provinces la Loi condamne un exterminateur pour tout effet néfaste résultant de ses actes, même s'il travaille pour le bien de la population.

\section{FAIRE LE RELEVÉ DE LA RÉGION EN ÉTÉ OU EN AUTOMNE}

Se procurer des cartes de la région, à l'échelle la plus grande possible, et les diviser en sections de grandeur appropriée, chacune d'elle identifiée par une lettre de l'alphabet. Examiner ensuite chaque section de terrain et indiquer sur la carte l'emplacement de tout endroit où l'eau séjourne ou risque de séjourner au printemps. Numéroter chaque foyer de reproduction à mesure qu'on l'inscrit sur la carte.
Éliminer tout de suite, si possible, les étangs ou les marais par de simples travaux de drainage ou de remblayage, ou noter l'emplacement pour les travaux de génie à entreprendre quand on aura les fonds nécessaires. Profiter de la maind'oeuvre disponible durant la saison morte pour aménager des sentiers jusqu'aux endroits d'accès difficile ou pour couper les broussailles. Les opérations du printemps s'en trouveront simplifiées.

\section{COMPTER LES LARVES AU DÉBUT DU PRINTEMPS}

Au printemps, dès que l'eau commence à séjourner, fouiller soigneusement le terrain pour établir où et quand les larves se développent. Inspecter chaque foyer possible de reproduction et voir s'il y a des larves. Pour cette observation, l'instrument le plus pratique est une louche émaillée d grand manche. Se rappeler que les larves de maringouins s'effraient à la moindre chose et plongent au fond de l'eau dès que l'on approche. Les laisser remonter à la surface, puis plonger la louche d'un coup rapide en dérangeant l'eau le moins possible. Noter le nombre de larves par prélèvement, de même que leur grosseur relative et voir s'il y a des nymphes. Lorsque l'étang est grand, faire plusieurs prélèvements à intervalles réguliers le long du rivage et inscrire les observations sur des cartes ou dans un carnet, en les reférant au numéro attribué à cet étang sur la carte topographique. Une trace de sabot ou une ornière peuvent offrir un refuge aux moustiques, aussi ne 
négliger aucune source possible. II peut se trouver de nouveaux foyers non indiqués sur la carte; les ajouter et leur assigner un numéro.

Les étangs profonds de nature permanente ou communiquant avec des cours d'eau sont en général exempts de maringouins à cause des poissons et des autres ennemis naturels des larves. Cependant, quelques-uns de ces étangs se réduisent par endroits en bas-fonds herbeux où les larves peuvent se mettre à l'abri de leurs ennemis naturels; il faut donc en inspecter chaque partie.

\section{OUTILLAGE}

II faut porter des vêtements protecteurs pour préparer et appliquer les produits chimiques. Les employés chargés des relevés ont en outre besoin de bottes de pêcheur. Les maringouins peuvent piquer a travers les vêtements aux endroits où ceux-ci sont étirés. Afin de donner une protection prolongée pulvériser un insectifuge sur les vêtements de coton ou de laine. Dans les circonstances extrêmes, recourir à un habillement protecteur: combinaison, gants, casque moustiquaire, mais l'inconfort de tout cet attirail par temps chaud lui confère une utilité fort douteuse.

Lire l'étiquette sur le récipient d'insecticide et suivre les précautions qui y sont énoncées.

Les pulvérisateurs ordinaires à bras (fig. 1) sont les plus simples pour l'application d'insecticides a l'intérieur des bâtiments ou dans des

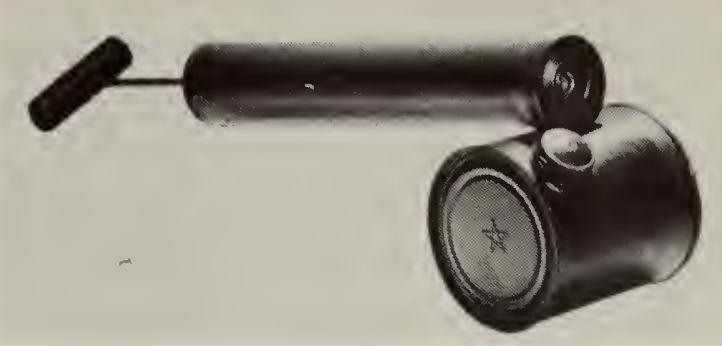

Figure 1. Pulvérisateur à bras.

récipients artificiels comme de vieilles boîtes en fer-blanc, des barils à huile ou des pneus usagés.

Les pulvérisateurs portatifs à air comprimé (fig. 2), portés sur l'épaule ou sur le dos peuvent servir pour le gros des arrosages à la main, effectués à l'extérieur. Ils ont une capacité de 1 à 5 gallons. Une pompe incorporée à l'appareil comprime l'air au-dessus de la solution d'insecticide, qui une fois relâchée, sort par un ajutage réglable suivant diverses formes de jet.

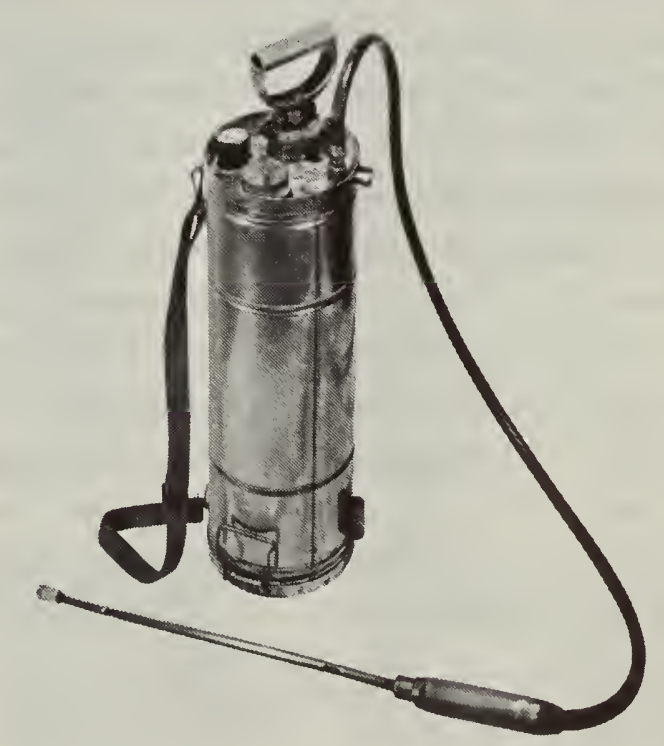

Figure 2. Pulvérisateur portatif à air comprimé. 
Les pulvérisateurs motorisés peuvent dispenser de grosses quantités de produits et leur jet a une portée plus longue que celui des pulvérisateurs à bras. Un modèle très utile (fig. 3) se porte sur le dos et se règle aussi bien pour l'application de poudre ou de granules que pour l'émission de brouillard ou de pluie. Les appareils motorisés (fig. 4) peuvent atteindre les dimensions du pulvérisateur à très grand travail, monté sur camion.

Dans les pulvérisateurs pneumatiques (ou nébulisateurs fig. 5) qui s'emploient autant pour la destruction des larves que pour celle des adultes, le liquide est injecté dans un puissant courant d'air orientable à volonté. Ces appareils varient, de ceux que l'on porte sur le dos, (fig. 3) à ceux qui contiennent plusieurs centaines de gallons.

Les générateurs thermiques d'aérosols également se trouvent en divers formats. Leur fonctionnement repose sur l'injection d'une suspension huileuse d'insecticide dans un courant d'air chaud ou de vapeur. II en résulte un fractionnement de l'huile en très fines gouttelettes qui flottent dans l'air et ne se déposent que très lentement. Les modèles les plus petits peuvent s'adapter au tube d'échappement des moteurs légers à refroidissement par air. D'autres appareils autonomes, portatifs, fonctionnent sur le principe de la pulsoréaction (fig. 6). Les grands appareils portés utilisent un brûleur à huile ou à essence pour chauffer le courant d'air et leur débit peut atteindre jusqu'à cent gallons à l'heure.

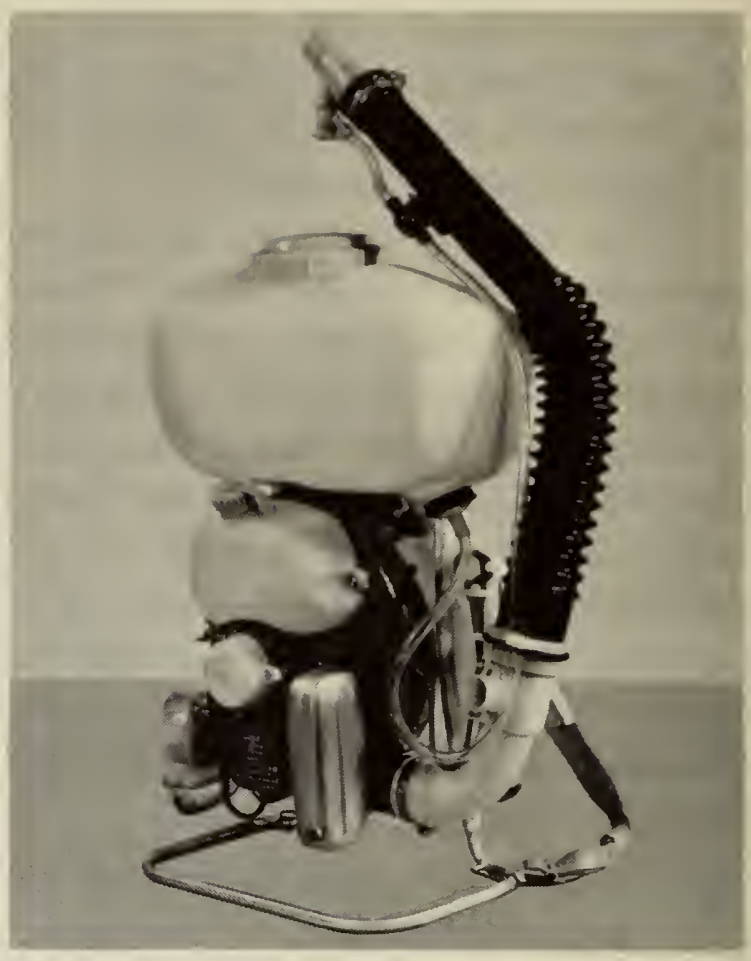

Figure 3. Pulvérisateur motorisé, porté à dos.

Les semoirs rotatifs à la main sont utiles pour épandre des granules insecticides sur de faibles étendues.

Les avions et les hélicoptères sont d'usage courant lorsqu'il s'agit de vaporiser ou de répandre des granules sur de vastes superficies. Leur emploi peut dispenser une municipalité ou une région, de mises de fonds considérables en matériel de surface: en effet ils ont l'avantage de couvrir une vaste étendue en peu de temps, et de plus ils peuvent survoler des terrains qui seraient trop accidentés ou trop mous pour la machinerie terrestre.

Les avions ont plusieurs avantages sur les hélicoptères: ils peuvent transporter jusqu'à 600 gallons d'insecticides, leur coût horaire d'opération est relativement peu élevé et ils sont très efficaces 


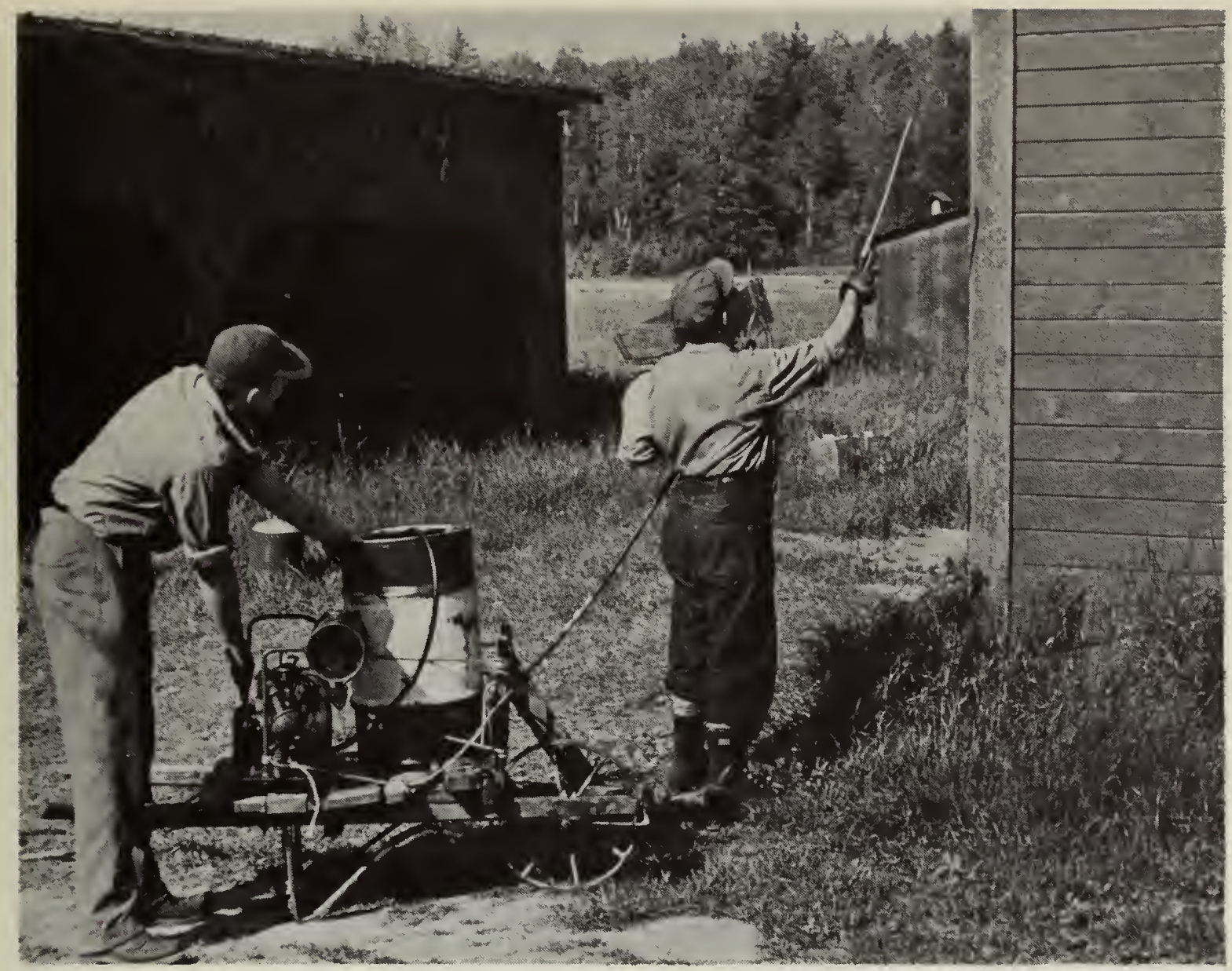

Figure 4. Petit pulvérisateur motorisé pour pulvérisations rémanentes.

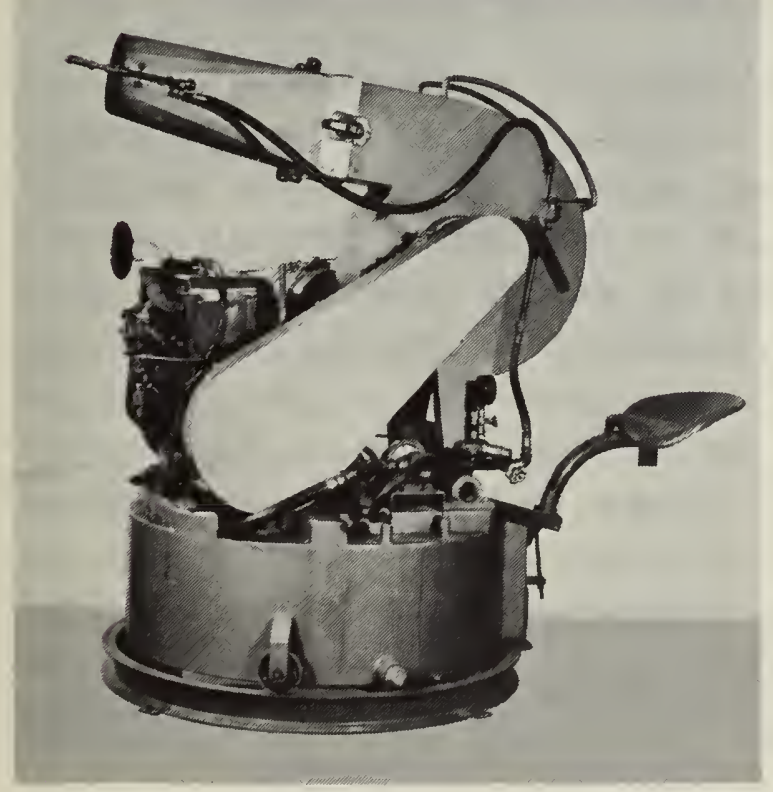

Figure 5. Pulvérisateur pneumatique. sur de grandes étendues de terrain uniforme. Par contre, leur emploi s'accompagne de certains inconvénients: si l'avion doit venir d'assez loin, il peut ne pas être disponible quand on en a le plus pressant besoin; les insecticides peuvent dériver jusque dans des régions qui ne doivent pas être contaminées;

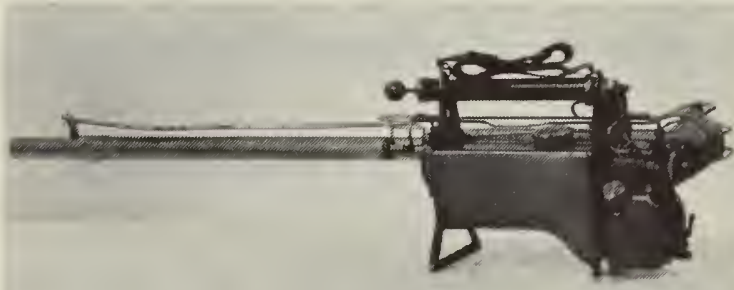

Figure 6. Générateur d'aérosol. 
les pulvérisations aériennes dépendent grandement des conditions atmosphériques, de sorte que le mauvais temps risque parfois d'interdire les traitements pendant plusieurs jours de suite.

Les frais d'opération des hélicoptères sont en général plus élevés que ceux des appareils à ailes fixes, leur charge utile est moindre et leur chargement prend plus de temps. Par contre leur supériorité apparaît dans le traitement de nombreux endroits isolés car ils peuvent placer le produit avec précision et ils ont l'avan. tage de pouvoir atterrir et recharger près des terrains à traiter.

Prévoir avec soin tous les préparatifs avant de retenir les services d'un avion. Aviser l'opé rateur assez tôt pour qu'il puisse s'arranger pour arroser en temps requis, car le temps est souvent un élément clé pour l'efficacité des traitements. Mettre au point des cartes indiquant jusque dans les détails les régions qui doivent être traitées et s'assurer que le pilote sait ce qu'il faut faire. Aménager à des endroits appropriés des installations de mélange et de chargement à moins que l'opérateur de l'avion ne s'en charge. Avoir des hommes disponibles pour baliser le terrain au besoin. Vérifier l'efficacité du traitement par des comptages de larves ou d'adultes avant et après l'application.

\section{ÉLIMINER LES FOYERS DE REPRODUCTION}

Les maringouins ne peuvent éclore qu'en eau dormante; aussi, la meilleure façon de les empêcher de se reproduire est de retirer toute l'eau où ils séjournent.

Certains foyers de reproduction seront assez faciles à supprimer; toutefois, une partie de ces travaux exigeront l'avis d'un ingénieur professionnel de même que des mises de fonds considérables. L'argent consacré à détruire les foyers de reproduction constitue, il est vrai, un excellent placement et une fois les travaux réalisés, leurs effets sont permanents. Un autre avantage est qu'au cours des traitements, on assainit en outre des terrains poten. tiellement productifs.

Vider ou enlever les récipients artificiels. Basculer les barils à huile sur le côté et transporter au dépotoir les vieilles boîtes en fer blanc et les vieux seaux. Vérifier les gouttières et nettoyer celles qui sont bouchées. Détruire les vieux pneus, car ils sont difficiles à assécher et si ce n'est pas possible, les trover en plusieurs endroits ou étendre leur talon avec un morceau de bois et les laisser sécher sur le côté.

Maintenir soigneusement désherbé le bord des canaux d'écoulement et bassins, où les larves pourraient se cacher: leurs ennemis naturels et les petites vagues suffiront alors d'ordinaire à les éliminer. Si une forte concentration de larves se formait, abaisser d'un pied environ le niveau de l'eau de façon à échouer les larves sur le rivage. II faut que la décharge dispose d'un canal sans obstruction jusqu'aux cours d'eau. Éviter la formation d'un marécage par les eaux d'infiltration.

Inspecter les arbres creux et les 
remplir de sable additionné au besoin d'un peu de poudre de DDT.

Drainer les marécages en creusant des fossés colateurs, combler les dépressions de terrain et élever des digues protectrices contre les inondations. La plupart des municipalités lovent la machinerie nécessaire à ce genre de travaux, ou encore les font exécuter à forfait. Seuls les groupements importants achètent leur propre machinerie de terrassement pour ces travaux d'assainissement.

$\mathrm{Ne}$ pas laisser l'excédent de l'eau d'irrigation séjourner sur le sol. Assécher le sol ou bien recueillir l'eau dans une citerne d'où on pourra ensuite l'utiliser de nouveau en la renvoyant, à l'aide d'une pompe sur le côté le plus élevé du champ.

La submersion de vastes étendues de terrain lors de la construction de barrages sur les grandes rivières devrait être soumise à un contrôle de niveau. Il est très important de prévoir ce contrôle lors de l'étude des systèmes modernes d'énergie hydroélectrique ou d'irrigation, si l'on ne veut pas s'exposer plus tard à des dépenses et des peines considérables pour la destruction des maringovins. En abaissant d'un pied ou deux à la fois le niveau des eaux endiguées, on tue presque toutes les larves qui se ramassent sur les bords peu profonds.

\section{TUER LES LARVES}

Si l'on n'a pas empêché les maringouins de se reproduire en éliminant les eaux stagnantes, on peut les attaquer au stade larvaire. Les larves ne vivent que dans l'eau, aussi ne peuvent-elles échapper si on les attrappe avant l'éclosion des adultes. Il est important de traiter les eaux de manière à empêcher les larves d'y vivre tout en évitant de tuer les poissons et les autres insectes dont ils se nourrissent.

Les traitements à base de carbamates et de composés organophosphorés sont très efficaces. Une fois leur effet atteint, ces produits dégénèrent rapidement et deviennent inoffensifs. L'emploi du DDT, du lindane, du chlordane et des produits organochlorés voisins est maintenant limité du fait qu'ils empoisonnent les autres catégories d'êtres vivants. L'application d'une pellicule d'huile ou de kérosène sur l'eau ne donne pas satisfaction, car souvent la pellicule ne s'étend pas complètement à la surface, ne réussit pas à pénétrer une couverture végétale épaisse, brûle le foin dans les prairies inondées, ou s'évapore si elle est trop mince.

Les produits chimiques appropriés pour le traitement de divers endroits sont mentionnés plus loin. Avant de s'engager dans des opé rations de grande envergure, deman. der à l'entomologiste provincial ou au ministère de la Santé publique quels produits peuvent s'employer dans la région. Des changements continuels se produisent dans le domaine des insecticides.

\section{TRAITEMENT AVANT L'ÉCLOSION DES OEUFS}

$\mathrm{Si}$, de par l'expérience passée, on connait l'emplacement de foyers de reproduction, appliquer au sol 
un produit d longue remanence avant le dégel du printemps. Cette méthode a souvent des avantages du fait qu'd ce temps de l'année la maind'oeuvre est moins rare.

Employer pour ces traitements du DDT, en granules, en suspension huileuse ou en poudre mouillable, au taux de une demi-livre à l'acre.

Avoir soin de ne pas contaminer les cours d'eau, les cultures ou les fourrages.

\section{TRAITEMENTS APRÈS LE DÉBUT DE L'ÉCLOSION}

Dès que les relevés à la louche révèlent le début de l'éclosion des oeufs, avertir immédiatement l'équipe chargée de la destruction des larves. Avant de traiter, attendre que les premières larves aient atteint leur quatrième mue, ce qui donne aux autres larves le temps d'éclore. Ainsi on pourra les tuer toutes ensemble. Toutefois, ne pas attendre que les nymphes fassent leur apparition, car elles sont beaucoup plus dures à détruire que les larves.

Inspecter les surfaces d'eau permanentes chaque semaine pour déceler, le cas échéant, les nouvelles éclosions, puis traiter comme suit: lorsque ces eaux ne se déversent pas directement dans des cours d'eau, employer du DDT en suspension huileuse ou en granules à la dose de un quart de livre a l'acre. S'il y a le moindre danger pour le poisson, utiliser alors du fenthion à raison de 1 à 2 onces a l'acre.
Les mares et les flaques isolées de pluie ou de neige fondue peuvent se traiter avec des capsules de gélatine contenant soit des pyréthrines, soit un mélange d'HCH (BHC) et de DDT. Chaque capsule a un rayon d'action de 750 pieds carrés.

Sur les surfaces d'eau où les herbes, les roseaux ou d'autres plantes empêchent le dépôt d'une pellicule huileuse, ou sur les prairies humides parsemées de petites dépressions de terrain, employer du fenthion a raison de 2 onces à l'acre ou de l'Abate à raison d'une once, soit en pulvérisation, soit en granules. Ne pas utiliser les capsules sur ces terrains. Quelques-uns des granules resteront pris sur la végétation mais on peut compter que la plupart descendront jusqu'à l'eau. Pour cette raison, ils sont particulièrement efficaces pour les traitements aériens d'une région densément boi sée.

Fonds de rivière à crue et autres régions qui s'égouttent dans les cours d'eau. Tant que l'eau monte au printemps, l'éclosion a peu de chance de se produire, elle commence après que la rivière a atteint son niveau le plus élevé. II y a des rivières dont le niveau monte et descend à plusieurs reprises, donnant lieu après chaque sommet a une nouvelle éclosion. Du fait que les plaines alluvionnaires des rivières sont en général très étendues et d'accès difficile par terre, le traitement aérien est d'ordinaire économique et efficace. Pulvériser au malathion à raison d'une demi- 
livre à l'acre, au fenthion, une once à l'acre ou à l'Abate, une demionce.

Les étangs d'épandage, de plus en plus nombreux au pays, peuvent constituer des foyers de reproduction gênants, si on ne les entretient pas comme il faut. On trouvera peut-être que des espèces grossières de poissons, comme la carpe, s'y plaisent et consomment toutes les larves qu'elles peuvent rejoindre. La plupart des larvicides dégénèrent rapidement dans ces étangs. Le fenthion, au taux de 2 onces à l'acre fait effet pendant quelques jours.

Rechercher et inspecter régulièrement tous les bassins d'ornementation, les tonneaux à pluie et à incendie. Traiter ceux qui contiennent des larves, par une pulvérisation légère de pyréthrine à 0.01 p. 100 en émulsion aqueuse, appliquée avec un pulvérisateur à bras ( 14 onces liquides par 100 pieds carrés). Le traitement ne sera peut-être pas nécessaire dans les bassins qui abritent des poissons.

Les bassins collecteurs des eaux de rue peuvent parfois produire beaucoup de maringouins: les arroser d'une demi-chopine de fenthion ou de nalède à 1 p. 100, mais il faudra répéter le traitement après chaque pluie abondante.

Les vastes étendues ou les endroits inaccessibles se traitent d'ordinaire plus économiquement par la voie des airs. Les traitements sont le plus souvent exécutés par des spécialistes commerciaux qui connaissent bien les techniques à suivre, de même que les restrictions légales qui pourraient être en vigueur dans telle ou telle localité. Ils peuvent donner des conseils sur le meilleur produit à employer dans une situation donnée et bien souvent ils le fourniront eux-mêmes.

\section{TUER LES MARINGOUINS ADULTES}

Dans les maisons. Les insecticides les plus pratiques pour l'usage domestique sont les aérosols du commerce vendus en bombes sous pression. Utiliser, aussi un mélange de DDT, de pyréthrine et de butoxyde de piperonyle qui s'achète tout préparé sous forme concentrée. Mélanger le concentré à du kérosène désodorisé, de façon à obtenir une concentration de $1 / 2$ p. 100 de DDT, et I'appliquer avec un petit vaporisateur à main (fig. 1), à la dose de une demi-once liquide pour un espace de 1,000 pieds cubes. Les fines gouttelettes se répandront dans la pièce et tueront au contact tous les maringouins. Le traitement ne détruit que les insectes présents à ce moment-là et si la maison n'est pas bien pourvue de moustiquaires, il faudra le répéter.

Ces aérosols sont très inflammables, aussi ne pas les employer dans les lieux où se trouvent des flammes.

Dans les grandes salles publiques et autres grands espaces fermés, employer le type le plus petit de générateur d'aérosol ou les plus grands pulvérisateurs à bras, avec les mêmes produits et la même dose que dans les maisons. 
Dans les étables laitières et les entrepôts. Vaporiser les surfaces intérieures d'une solution aqueuse de nalède à 1 p. 100 en grosse pluie, de façon à laisser un résidu. Se servir d'un pulvérisateur à bras ou à air comprimé, à la dose de 2 gallons de solution par 1,000 pieds carrés. Si l'on recherche une protection durable dans des endroits fermés où l'air circule peu, utiliser les bandes au dichlorvos suspendues au plafond, elles libèrent assez de vapeur pour tuer mouches et moustiques pour plusieurs mois. On peut les employer dans les maisons, mais il faut bien observer les précautions indiquées sur l'étiquette.

Vastes espaces d l'extérieur. Arroser au malathion en solution huileuse à raison de 2 à 8 onces dे l'acre, ou au fenthion à 2 onces a l'acre. Les travaux sont d'ordinaire effectués par des entrepreneurs, avec des avions.

Les aérosols thermiques, utilisés à bon escient, constituent un moyen très efficace de traiter un espace extérieur à partir du sol et ont l'avantage de ne laisser aucun résidu sur les plantes.

Ces aérosols, ou brouillards, peuvent être relâchés par toute une gamme de générateurs, depuis les petits modèles que I'on adapte au tube d'échappement des tondeuses à gazon jusqu'aux machines à très fort débit montées sur camion. Employer du DDT à 5 p. 100, du malathion à 3 p. 100 ou une suspension de nalède à 1 p. 100 dans de l'huile à carburant no 2. Pour couvrir une région, mettre le générateur en marche et le tirer lentement (à 4 ou 5 milles à l'heure) perpendiculairement à la direction du vent, en une série de lisières parallèles. Les grosses machines sont efficaces pour 100 à 200 verges sous le vent et les plus petites ont des rayons d'action réduits en proportion. En terrain découvert, un vent de 2 à 4 milles à l'heure est idéal, mais dans les bois on aura les meilleurs résultats avec un vent de 6 à 10 milles a l'heure (au-dessus des arbres).

On ne devrait recourir aux générateurs d'aérosols que lorsqu'il se produit un renversement de température dans l'atmosphère, c'estd-dire quand la fumée des cheminées s'accroche au sol plutôt que de monter vers le ciel. Le phénomène, d'ordinaire, précède le crépuscule et se prolonge peu après l'aube, mais on l'observe parfois par temps couvert. En pareilles circonstances, le brovil. lard d'aérosols se trainera au sol, tandis que par ciel clair ou s'il vente, il s'élèvera pour se dissiper.

Les pulvérisateurs pneumatiques peuvent servir au même usage mais les gouttelettes qu'ils produisent sont plus grosses que celles des générateurs d'aérosols et retombent au sol. Avec ces appareils traiter en lisières plus rapprochées et ne pas employer de DDT près des terres cultivées. L'efficacité du pulvérisateur pneumatique ne dépend pas autant de la vitesse et de la direction du vent que celle des générateurs d'aérosols, l'opérateur étant en mesure d'orienter le jet où il le désire jusqu'à une certaine distance. 
Espaces extérieurs isolés. Traiter une ceinture de végétation de 20 à 50 pieds de large sur le pourtour de la zone à protéger, par pulvérisation de DDT à 5 p. 100, en suspension de poudre mouillable, iusqu'à une hauteur de 10 pieds. Ensuite traiter avec des aérosols l'espace ainsi encerclé. La ceinture retardera de nouvelles invasions de l'extérieur, mais il faudra répéter au besoin le traitement aux aérosols. Les endroits où les maringouins se posent, comme les clôtures et les abords des bâtiments pourront recevoir un traitement rémanent par pulvérisation d'une solution huileuse de DDT à 5 p. 100, à raison de 1 gallon par 1,000 pieds carrés.

Ne pas employer les solutions huileuses de DDT sur les plantes ef éviter le DDT pour celles qui doivent servir de fourrage.

\section{POUR MESURER LE SUCCÈS DE LA CAMPAGNE}

Pour être à même d'apprécier le degré de réussite de la campagne, estimer l'importance de la population de maringouins adultes plus tard durant l'été. Les moyens les plus simples consistent à effectuer des comptages d'atterrissages, de piqûres, ou des comptages au filet ou aux pièges lumineux. Les comptages devront se répéter aux mêmes endroits et à intervalles réguliers, de manière à pouvoir suivre les hausses ou les baisses de la population. Veiller à occuper les postes de comptage à la même heure chaque fois; effectuer des comptages en dedans comme en dehors de la zone traitée, autant que possible à peu près en même temps; on aura ainsi une idée de l'efficacité des traitements.

Comptage des maringouins: rester-immobile une minute environ, puis compter les maringouins qui se posent, en une minute, entre la taille et les genoux. Recommencer trois fois, à une minute d'intervalle chaque fois, puis faire la moyenne et l'inscrire pour le poste en question.

Comptage des piqûres; exposer un bras nu pour une minute et compter les piqûres subies pendant ce temps entre le coude et le poignet. Recommencer trois fois et faire la moyenne.

Le comptage à la volée exige l'emploi d'un filet ordinaire a insectes. Une fois en place, laisser s'apaiser le déplacement d'air, puis prendre dix coups de filet à bout de bras et compter les maringouins capturés. S'il faut recommencer, le faire toujours de la même façon.

Les pièges lumineux peuvent aussi servir à l'échantillonnage des populations adultes. En Amérique du Nord, les préposés à la lutte emploient le piège régulier New Jersey, qui comprend une source de lumière pour attirer les insectes, un ventilateur pour les aspirer et un bocal à poison pour les tuer. Tout comme dans les autres modes de comptage, un piège utilisé au même endroit à intervalles réguliers. mesurera les hausses ou les baisses de la population a cet endroit, alors que des pièges disposés en divers endroits mais au même moment 
permettront de comparer les densités de population.

\section{MESURES PRÉVENTIVES DANS LES MAISONS ET LES TENTES}

Si l'on n'a pas réussi à éliminer les maringouins à l'extérieur, équiper les portes et fenêtres des maisons de moustiquaires. Les mailles devraient avoir au minimum $16 \times 16$ au pouce carré. Quoique plus chers à l'achat, les moustiquaires en matériaux anti-rouille comme le fil galvanisé, le cuivre ou le plastique feront plus que compenser la dépense supplémentaire. Garder les portes moustiquaires bien ajustées et en bon état.

Au camping, employer une tente munie d'un tapis de sol cousu et d'un volet supplémentaire en moustiquaire que l'on peut fermer hermétiquement. Avant de se coucher, tuer les maringouins présents dans la tente avec une bombe aérosol, puis fermer la moustiquaire.

\section{INSECTIFUGES}

Les insectifuges sont utiles pour ceux qui ont des occupations au grand air mais la durée de la protection qu'ils apportent varie selon les circonstances. De nos jours, une seule application d'un bon insectifuge soulagera, pour presque toute la journée, un promeneur peu pressé. Par contre un homme qui s'adonne à des exercices ou des travaux violents doit s'en mettre toutes les heures ou presque.

II existe tout une gamme de marques diverses de produits insectifuges, soit en liquide, en crème ou en pulvérisation. Parmi les ingrédients actifs qu'ils renferment le meilleur à l'heure actuelle est le diéthyl toluamide, quoique le diméthyl phthalate et l'éthyl hexanediol soient très bons. D'autres produits prometteurs sont à l'essai et seront peut-être bientôt en vente.

En appliquant l'insectifuge sur la peau, éviter d'en mettre sur les yeux ou sur les lèvres. Tous les insectifuges sont des solvants des plastiques et peuvent donc endommager les montures de lunettes, les verres de montre et les tissus synthétiques comme le nylon.

Pour de plus amples renseignements, écrire à la Division de l'information, ministère de l'Agriculture du Canada, Ottawa, Canada. 


On peut obtenir des exemplaires de cette publication à la: DIVISION DE L'INFORMATION

\section{MINISTÈRE DE L'AGRICULTURE DU CANADA OTTAWA}

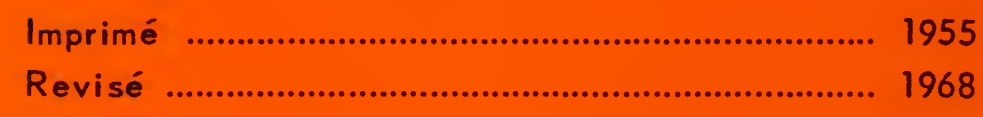

ROGER DUHAMEL, MSAC, IMPRIMEUR DE LA REINE ET CONTRÔLEUR OE LA PAPETERIE, OTTAWA, I968 